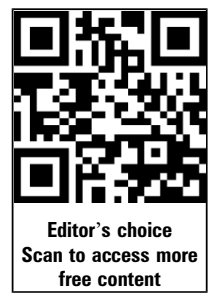

${ }^{1}$ Department of Pediatrics, Hospital Medicine, University of California, San Francisco, San Francisco, California, USA ${ }^{2}$ School of Nursing, UCSF, San Francisco, California, USA ${ }^{3}$ The Permanente Medical Group, Oakland, California, USA ${ }^{4}$ Department of Medicine, UCSF, San Francisco, California, USA

\section{Correspondence to}

Dr Glenn Rosenbluth, Department of Pediatrics, Hospital Medicine, University of California, San Francisco, 550 16th St, Mail Code 3214, San Francisco, California 94143 USA; glenn.rosenbluth@ucsf.edu

Received 10 July 2015 Revised 13 October 2015 Accepted 20 October 2015 Published Online First

11 November 2015

\section{SLLinked}

- http://dx.doi.org/10.1136/ bmjqs-2015-005027

\section{CrossMark}

To cite: Rosenbluth $G$, Jacolbia R, Milev D, et al. BMJ Qual Saf 2016;25:324328.

\title{
Half-life of a printed handoff document
}

\author{
Glenn Rosenbluth, ${ }^{1}$ Ronald Jacolbia, ${ }^{2}$ Dimiter Milev, ${ }^{3}$ \\ Andrew D Auerbach ${ }^{4}$
}

\begin{abstract}
Background Printed handoff documents are nearly universally present in the pockets of providers taking inhouse call. They are frequently used to answer clinical questions. However, the static nature of printed documents makes it likely that information will quickly become inaccurate as a result of ongoing management. This increases the potential for medical errors, especially in clinical services which rely heavily on printed documents for ongoing patient management.
\end{abstract}

Objective To measure the average time to potential inaccuracy, represented as the 'half-life' of printed handoff documents.

Design, setting, participants Cross-sectional analysis of 100 adult inpatients during a single $24 \mathrm{~h}$ period at an academic medical centre in 2014.

Main outcome and measure The half-life was defined as the time at which half of the patients would be expected to have inaccurate information on a printed handoff document, based on review of orders which populate data fields on these printed handoff documents.

Results In our sample, the half-life was $6 \mathrm{~h}$ on the $12 \mathrm{~h}$ night shift and $3.3 \mathrm{~h}$ on the day shift. We identified at least on change within the $24 \mathrm{~h}$ period for $92 \%$ of patients. Most changes (90\% $\mathrm{n}=1411$ ) were medication-related, but the overall distribution of order types was significantly different between day and night ( $p=0.002)$.

Conclusions and relevance The accuracy of printed handoff documents quickly deteriorated over the course of a physician shift. Based on this decay rate, a typical physician getting signout on 20 patients overnight can safely assume that the data for 10 of them will be inaccurate or outdated in $6 \mathrm{~h}$ and that it will be inaccurate on another two by the morning.

\section{INTRODUCTION}

Medical errors related to poor communication remain unacceptably common. ${ }^{1}$ Verbal handoffs are known to be highrisk communication, and best-practice guidelines and recommendations for verbal handoffs have been published. ${ }^{2-5}$ Among these recommendations are that communication should be face-to-face, and a printed document should accompany the verbal handoff to both ensure completeness and to provide additional information as a reference. Efforts to improve printed and verbal handoffs have been associated with reductions in medical errors and adverse events. ${ }^{6-8}$ In spite of recommendations and bestpractice guidelines, providers may still rely exclusively on printed handoff documents without a verbal handoff. ${ }^{9}$

Much less is known about best practices for printed handoff documents, despite their near-universal presence in the pockets of providers taking inhouse call, and their frequent usage to answer clinical questions. ${ }^{10-12}$ Printed documents typically contain clinical information about patients for whom the physicians are responsible for. They serve as references when answering questions during shifts, either in isolation or together with other resources. ${ }^{11}$ In addition, physicians may use the printed documents for other purposes, including as patient census lists, references for oral presentations on rounds and daily workflow documents.

The static nature of printed documents makes it likely that some of the information will quickly become inaccurate, increasing the potential for medical errors. Put simply, depending on the data elements included, the paper document has the potential to be outdated a moment after it is printed. Outdated information may have limited impact in settings where the providers are very familiar with the patients, such as a primary intern on a teaching service who uses the document as a reference for presenting on rounds. However, another team member (eg, senior resident) could 
easily commit an error if $\mathrm{s} / \mathrm{he}$ used the document as a reference without knowing that the intern had changed orders after rounds.

Computerised handoff documents integrated with electronic health records (EHR) have been associated with improvements, ${ }^{13}$ including perceived accuracy $^{14} 15$ and rounding and sign-out processes. ${ }^{16}$ However, in these studies of computerised handoff templates the integrated document was still printed. ${ }^{14-16}$ Printing makes real-time automatic updating impossible, and therefore, increases the potential for inaccurate information. In principle, the recommendations above regarding 'printed documents' (as accompaniments to verbal handoffs) could refer to either on-screen or a paper documents. However, we could find no data to describing changes in printing of documents after implementation of EHR, nor could we identify any studies of exclusively on-screen handoff documents.

In this pilot study we calculated the median time to inaccuracy, referred to as the 'half-life,' of printed handoff documents. Our goals were to determine the time at which half of the patients on a clinical team were likely to have at least one printed inaccuracy, to characterise the types of inaccuracies, and to identify differences between day and night shifts, as well as surgical and non-surgical services. We hypothesised that by the end of an overnight call shift, most handoff documents would contain at least one error, which had the potential to impact patient care. We used the term half-life and displayed the data as a survival curve because we felt this model would resonate with physicians.

In academic medical centres, including ours, most residents print handoff documents-often at the start of a work shift or work day-then refer to them during the ensuing time. However, our results suggest that a potentially important number of printed elements may become inaccurate soon after the static printout is obtained. By documenting the inaccuracies which can be expected on printed handoff documents, we hope to achieve a shift toward reliance on the EHR (on-screen, real-time) as the 'source of truth,' with the ultimate desired result of improved patient safety.

\section{METHODS}

\section{Study site}

Our study was conducted at a quaternary care academic medical centre. Our institution's primary handoff document is generated from our EHR. The document includes common fields, which are autoimported from administrative data (eg, patient location, pharmacy-generated medication lists). The handoff document also includes fields such as patient summary, which are autoimported from physicians' free-text entries elsewhere in the EHR. Individuals have the option to view the document on-screen and/ or print at workstations throughout the hospital.

\section{Study population}

We generated a list of all inpatient admissions during a randomly selected $24 \mathrm{~h}$ period. We ordered this list by medical record number, and identified the first 100 patients, excluding paediatric patients as well as patients on the labour and delivery, and intensive care unit services. Patients were on clinical services, cared for using a variety of staffing models, including residents, fellows, attendings and/or nurse practitioners.

\section{Data collection/analysis}

We identified a set of data fields commonly included in printed handoff documents at our institution and many similar institutions: medications, diet, code status, and patient location. These fields were selected because they could reasonably be expected to change during a $24 \mathrm{~h}$ period, changes could be objectively measured by reviewing orders and could be reasonably expected to impact care. We reviewed all orders written on subjects within a $24 \mathrm{~h}$ period to identify those orders which would potentially change the data in those fields. We documented order type, time and identity of the order-writer. We arbitrarily defined the start of our $24 \mathrm{~h}$ period as 18:00, the approximate time at which a cross-covering physician would print a handoff document for a night-shift.

We calculated the time to first order for each patient, as this would be the time to first potential inaccuracy on a printed handoff document. We then plotted the data for all changes as a survival curve showing the per cent of patients with correct (unchanged) data over time. This enabled us to determine incidence of inaccurate information on a theoretical handoff document for these patients, at various time points after printing. The 'half-life' was the time at which half of the patients would have had inaccurate information on a printed handoff document, equivalent to the median time to inaccuracy. Comparisons were made between a $24 \mathrm{~h}$ shift starting at 18:00 (common time of handoff), $12 \mathrm{~h}$ day (06:00-17:59), and night shifts (18:00-05:59), as well as between medical and surgical services.

Statistical analysis was performed using t-tests to compare the mean half-life time observed between groups and $\chi^{2}$ tests to compare the distribution of order types observed between groups. All analyses were conducted using $\mathrm{R}$ data analysis software.

The study was reviewed and approved by the UCSF Committee on Human Research.

\section{RESULTS}

We reviewed 1563 total orders on 100 patients. Patient demographics are shown in table 1 . The medical services (including neurology) accounted for 56 patients, and the surgical services accounted for 44 
Table 1 Demographics of subjects

\begin{tabular}{lc}
\hline Summary of demographics & $\mathrm{N}=100$ \\
\hline Service & \\
Surgical & 44 \\
Non-surgical & 56 \\
Length of stay (in days) mean (SD) & $21(27)$ \\
Age (in years) mean (SD) & $60(14)$ \\
Gender & \\
Female & 51 \\
Male & 49 \\
Race & \\
American-Indian or Alaska native & 1 \\
Asian & 10 \\
Black or African-American & 9 \\
Native Hawaiian or other Pacific islander & 1 \\
Other & 15 \\
Unknown/declined & 1 \\
White or Caucasian & 63 \\
Ethnicity & \\
Hispanic or Latino & 13 \\
Not Hispanic or Latino & 87 \\
Insurance & \\
Medicare & 22 \\
Medicaid & \\
Private & \\
Other & 21 \\
\hline & \\
\hline
\end{tabular}

patients. At least one order was written on the night shift for $59 \%$ of the patients, and at least one order was written on the day shift for $86 \%$. Over the full $24 \mathrm{~h}$ period, $92 \%$ had at least one order. Most orders (90\%) were medication related on both day and night shifts. The overall distribution of order types was significantly different between day and night, with more code-status orders written at night and more diet orders written during the day $\left(\chi^{2} \mathrm{p}=0.002\right)$.

The half-life of the printed handoff document on the $12 \mathrm{~h}$ night shift $(18: 00-06: 00)$ was $6 \mathrm{~h}$ (figure 1 ) and on the $12 \mathrm{~h}$ daytime shift was $3.3 \mathrm{~h}$ (figure 2). The mean number of changes per patient was not different between shifts ( 6.8 on night shift, 8.8 on day shift, ns). There were significantly more unique providers writing orders on each patient on the day shift compared with the night (2.5 vs 1.4 on providers per patient, $\mathrm{p}=0.0004$ ).

Surgical patients had similar average total orders per patient (18.2 vs 13.4 on non-surgical, ns), similar number of order-writing providers per patient (3.6 vs 3.1 on non-surgical, ns), and a much lower half-life duration $(2.2 \mathrm{~h}$ vs $6 \mathrm{~h})$. There was a statistically significant difference between average time of first order change between the surgery and non-surgery cohorts (6.37 vs $8.98, \mathrm{p}=0.0501$ ).

\section{DISCUSSION}

Content in our modeled printed handoff documents quickly became out of date over the course of a physician shift. A typical resident getting sign-out on 20 patients overnight could safely assume that the data for 10 of them would be inaccurate or outdated in $6 \mathrm{~h}$, and that it would be inaccurate on another two by the morning. While our study did not attempt to discern the potential harms posed by these discrepancies, any changes to code status, diet, medications, or other orders could pose significant safety problems.

Although teams may be perceived to be working together, our data demonstrate that there is often more than one order-write per shift. In a highfunctioning team with good communication, this would not be a problem. However, one can easily imagine a single care provider interpreting clinical information without knowing that another team member had made a change, but forgot to tell others (eg, consider an attending who is informed of a new fever in a patient on whom the intern had discontinued antibiotics). Unless all care providers are immediately alerted to all changes, it is highly likely that at least some providers would be carrying documents with inaccurate data. Good communication practices, including briefs, huddles and clearly defined roles ${ }^{17}$ can increase the likelihood that all team members will have updated information after key events, such as code status discussions.

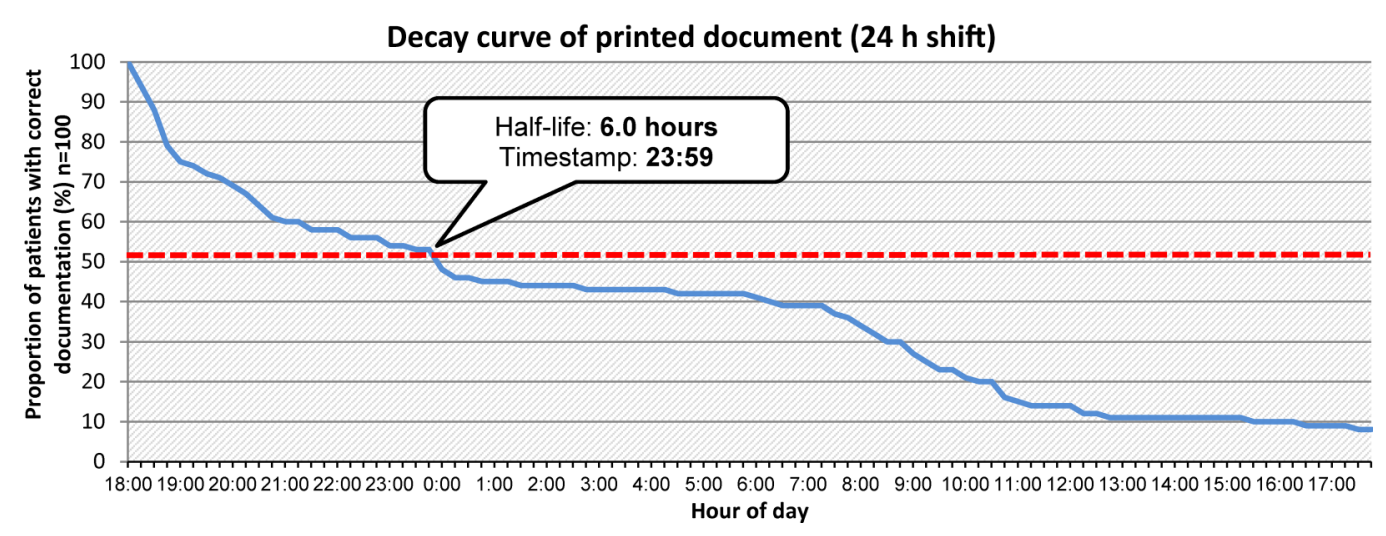

Figure 1 Half-life of printed handoff document, for $24 \mathrm{~h}$ period starting at 18:00. 


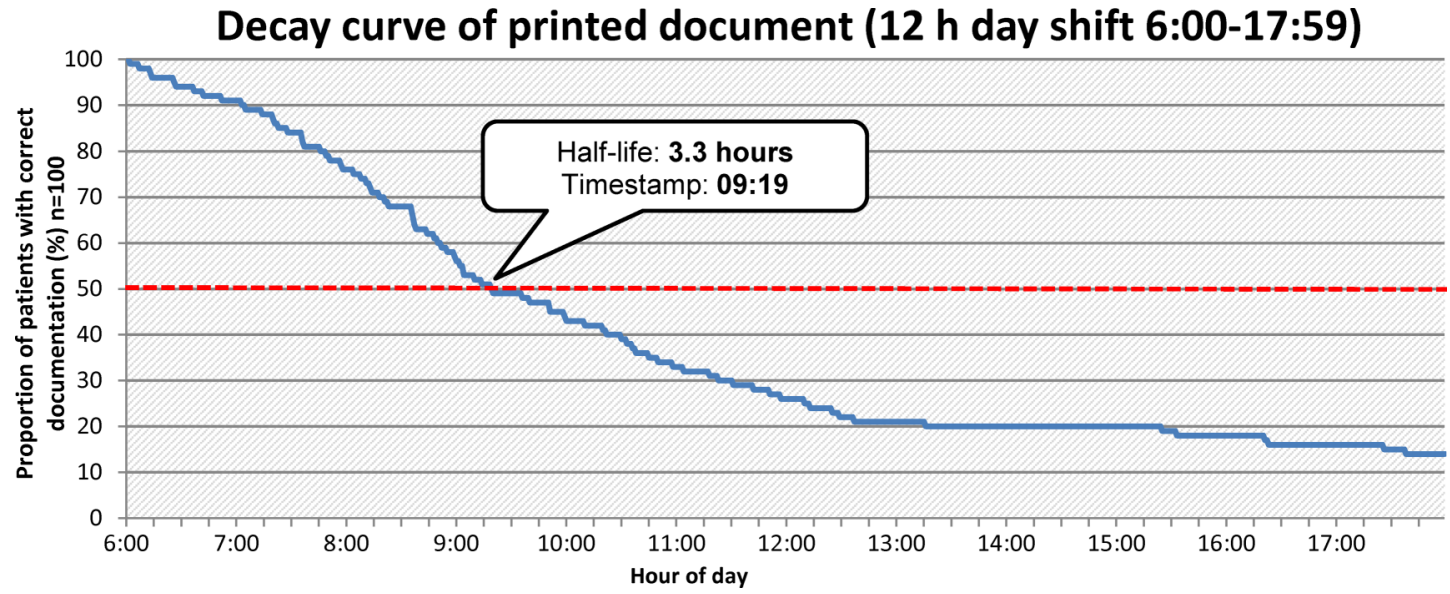

Figure 2 Half-life of printed handoff document, for $12 \mathrm{~h}$ period starting at 06:00.

Minimising the printing of documents would drive providers to look at information which is updated in real time, such as EHR-based medication lists and talking to nurses. Based on the patterns of change in our sample, the majority of which were medication-related, one could reasonably question the risk-benefit of including medication lists in printed handoff documents. This study and others ${ }^{18}$ suggest a need to further explore this area. Minimising printing would also decrease other risks, such as privacy-related violations.

We observed that potential discrepancies took place more frequently during daytime hours, likely due to active management as well as multiple order writers. This would have greater impact on those individuals who use handoff documents as a daily workflow document. Although the order-writers themselves may be aware of individual changes, other team member's documents would still need to be updated. This is particularly relevant for providers who may print a document early in the morning, and then be in procedures or clinics for most of the day.

Interestingly, code status changes, which would be critical to communicate due to the severe implications of miscommunications, were more common at night. Providers should be aware of the structural differences between day shifts, when teams may have a larger number of inhouse providers but with scheduled communication (eg, rounds), contrasted with night shifts, when teams may have only 1-2 providers inhouse, but without regularly scheduled communication.

Beyond the strategy of decreased printing, our work presents several strategies for workflow improvements to reduce the risk of medical errors due to inaccurate handoffs. Explicit identification of high-stakes decisions can aid in safe decision making. Providers should be taught to identify situations in which their medical decision making would be changed based on key pieces of information, which should then be verified in the EHR rather than the printed document. High functioning teams have clearly identified roles, ${ }^{17}$ and perhaps order writing could be such a role. Individual teams could funnel orders through a single order writer who would then be responsible for ensuring that the appropriate team members are aware and the handoff documents are updated.

Our study has several important limitations. Our sample is from a single institution during a single $24 \mathrm{~h}$ period. However, we included a variety of medical and surgical services, which increases the generalisability. As this was a pilot study, we have a relatively small number of patients from any individual service. Our sample is from a quaternary care academic medical centre, and therefore, patients may be sicker than the average hospitalised adult, limiting generalisability.

\section{CONCLUSION}

In this report, we identify a very high potential for inaccurate information in printed handoff documents. If a handoff document is printed at the start of a night shift, it is reasonable to assume that within $6 \mathrm{~h}$ the document will contain inaccuracies on half of the patients. The field most likely to contain inaccuracies is the medication lists, followed by code status. On day shifts, inaccuracies may accumulate even more quickly, likely due to active management as well as multiple order writers. Easy access to and usage of online handoff reports contained in EHR present a potential solution to this problem.

Correction notice This article has been corrected since it was published Online First. Figure 1 title has been corrected.

Twitter Follow Glenn Rosenbluth at @grosenbluth

Contributors All authors meet the following criteria: substantial contributions to the conception or design of the work or the acquisition, analysis or interpretation of data. Drafting the work or revising it critically for important intellectual content. Final approval of the version published. Agreement to be accountable for all aspects of the work in ensuring that questions related to the accuracy or integrity of any part of the work are appropriately investigated and resolved.

Funding National Institutes of Health (K24HL098372). 
Competing interests None declared.

Ethics approval UCSF Committee on Human Research. Provenance and peer review Not commissioned; externally peer reviewed.

\section{REFERENCES}

1 The Joint Commission. Sentinel Event Data-Root Causes by Event Type. 201 (cited 13 April 2015); http://www. jointcommission.org/assets/1/18/Root_Causes_by_Event_Type_ 2004-2Q 2014.pdf

2 Patterson ES, Roth EM, Woods DD, et al. Handoff strategies in settings with high consequences for failure: lessons for health care operations. Int J Qual Health Care 2004;16:125-32.

3 Vidyarthi AR, Arora V, Schnipper JL, et al. Managing discontinuity in academic medical centers: strategies for a safe and effective resident sign-out. J Hosp Med 2006;1:257-66.

4 Horwitz LI, Moin T, Green ML. Development and implementation of an oral sign-out skills curriculum. J Gen Intern Med 2007;22:1470-4.

5 Arora VM, Manjarrez E, Dressler DD, et al. Hospitalist handoffs: a systematic review and task force recommendations. J Hosp Med 2009;4:433-40.

6 Petersen LA, Orav EJ, Teich JM, et al. Using a computerized sign-out program to improve continuity of inpatient care and prevent adverse events. Jt Comm J Qual Improv 1998;24:77-87.

7 Starmer AJ, Spector ND, Srivastava R, et al. Changes in medical errors after implementation of a handoff program. N Engl J Med 2014;371:1803-12.

8 Starmer AJ, Sectish TC, Simon DW, et al. Rates of medical errors and preventable adverse events among hospitalized children following implementation of a resident handoff bundle. JAMA 2013;310:2262-70.
9 Schoenfeld AR, Salim Al-Damluji M, Horwitz LI. Sign-out snapshot: cross-sectional evaluation of written sign-outs among specialties. BMJ Qual Saf 2014;23:66-72.

10 Hanson JT, Leykum LK, Pugh JA, et al. Nighttime clinical encounters: How residents perceive and respond to calls at night. J Hosp Med 2015;10:142-6.

11 McSweeney ME, Landrigan CP, Jiang $\mathrm{H}$, et al. Answering questions on call: pediatric resident physicians' use of handoffs and other resources. J Hosp Med 2013;8: 328-33.

12 Fogerty RL, Schoenfeld A, Salim Al-Damluji M, et al. Effectiveness of written hospitalist sign-outs in answering overnight inquiries. J Hosp Med 2013;8:609-14.

13 Li P, Ali S, Tang C, et al. Review of computerized physician handoff tools for improving the quality of patient care. J Hosp Med 2013;8:456-63.

14 Palma JP, Sharek PJ, Longhurst CA. Impact of electronic medical record integration of a handoff tool on sign-out in a newborn intensive care unit. J Perinatol 2011;31: 311-17.

15 Wayne JD, Tyagi R, Reinhardt G, et al. Simple standardized patient handoff system that increases accuracy and completeness. J Surg Educ 2008;65:476-85.

16 Van Eaton EG, Horvath KD, Lober WB, et al. A randomized, controlled trial evaluating the impact of a computerized rounding and sign-out system on continuity of care and resident work hours. J Am Coll Surg 2005;200: 538-45.

17 US Department of Health and Human Services AfHRaQ. TeamSTEPPS home. (cited 8 June 2015); http://teamstepps. ahrq.gov/

18 Arora V, Kao J, Lovinger D, et al. Medication discrepancies in resident sign-outs and their potential to harm. J Gen Intern Med 2007;22:1751-5. 